\title{
La escolaridad de los padres como modelo a seguir en los estudiantes de traducción
}

\section{Parental schooling as a role model for translation in translation students}

\author{
DOI: $10.46932 / \mathrm{sfjdv} 2 \mathrm{n} 4-014$
}

Received in: May 1st, 2021

Accepted in: Jun 30th, 2021

\section{José Cortez-Godínez}

Doctor en Estudios Avanzados de Traducción e Interpretación (Universidad de Granada); Licenciado en Traducción del Idioma Inglés (UABC); Investiga la evaluación de la competencia traductora y sus subcompetencias. Imparte cursos de tecnología aplicada a los procesos de traducción. Responsable del Centro de Educación Abierta y a Distancia en la Facultad de Idiomas, Campus Mexicali.

Correo electrónico: jose_cortez@uabc.edu.mx

\section{RESUMEN}

En esta comunicación, resultado de un estudio exploratorio longitudinal finalizado en 2013, registramos cómo el grado de estudios de los padres de los estudiantes de traducción "modela" sus aspiraciones profesionales y la adquisición de estatus dentro de la sociedad. Los sujetos, en su desarrollo académico buscan emular al menos el grado de estudio de aquellos, o superarlo. Durante la medición de las variables, de una matriz adaptada, de la competencia traductora (PACTE 2000, 2001, 2003, 2005, 2008, 2009, 2011, 2014 y 2019), en los estudiantes del nivel intermedio de la Facultad de Idiomas-Mexicali, México, descubrimos que existe una correlación cuantitativa entre el estatus educativo de los padres (con grado universitario o mayor) y las futuras aspiraciones de desarrollo profesional de los estudiantes de traducción. En el proceso, encontramos que el nivel universitario en alguno de los padres influye en los estudiantes de manera positiva como motivación extrínseca, modelando sus deseos de autorrealización y estatus. El fenómeno, lo caracteriza Bandura (1987) como el comportamiento que se aprende del medio ambiente a través de un proceso de aprendizaje observacional y creemos, que si se establece esta influencia, se pueden buscar mecanismos de apoyo para reforzar el desarrollo de la competencia traductora en el aula por parte del instructor.

Palabras clave: Cultura, educación, competencia traductora, motivación extrínseca, modelos.

\begin{abstract}
In this communication, which is the result of a longitudinal exploratory study completed in 2013 , we record how the parents of translation students model them in their professional aspirations and the acquisition of status within society. The subjects, in their academic development, seek to emulate at least their parents' level of education, or surpass it. The measured variables are part of an adapted matrix of the translation competence (PACTE 2000, 2001, 2003, 2005, 2008, 2009, 2011, 2014, and 2019). During the applied procedures to the intermediate level students of the Language School-Mexicali Campus, Mexico, we found that there is a quantitative correlation between the educational status of the parents (with a university degree or higher) and the future aspirations for professional development of the translation students. In the process, we found that a parent's university level influences students positively as an extrinsic motivation, shaping their desires for self-realization and status. The phenomenon is characterized by Bandura (1987) as behavior that is learned from the environment through a process of observational learning. We believe that if this influence is established, support mechanisms can be sought to reinforce the development of translation competence in the classroom by the instructor.
\end{abstract}

Keywords: Culture, education, translation competence, extrinsic motivation, modeling. 


\section{INTRODUCCIÓN}

La situación espacio-temporal en la que se desenvuelve nuestro objeto de estudio (los alumnos de V semestre de la carrera de Licenciado en Traducción de la Facultad de Idiomas, Campus Mexicali), es una región fronteriza bicultural que tiene vecindad geográfica con Estados Unidos de América (EE.UU.), donde un alto porcentaje del español que hablamos, es inglés castellanizado (préstamos léxicos conjugados o flexionados al castellano, que rápidamente se integran al habla cotidiana del habitante de la frontera Basich (2012). Es la llamada cultura transfronteriza, y es dentro del ámbito de la cultura, donde la psicología se ha interesado en su influencia en el desarrollo cognitivo del individuo, y de cómo el aprendizaje (en el entorno familiar, social y en el aula) es el vehículo para interiorizar comportamientos, actitudes, valores y modelos a seguir, en este caso los transmitidos por los padres. "Esto significa que el aprendizaje de un nuevo concepto (en el aula, por ejemplo) sólo es posible si se establece alguna conexión con un concepto que ya está a disposición de quien aprende" (Smith, 2000, p. 154). La formación de nuestros estudiantes, al menos hasta su entrada a preescolar, es moldeada por padres, entorno familiar y social, por lo que los modelos a seguir por antonomasia son los propios padres.

Conocido es el viejo debate entre las teorías de Piaget-Vygotsky, sobre si la herencia y ambiente dan forma o determinan la mente del sujeto, sin embargo, ambos en mayor o menor grado, le dan crédito al entorno social ya sea como una "escalera" (Piaget) o como una "red" (Vygotsky): la zona de desarrollo próximo (ZDP) del sujeto no evoluciona en solitario (Smith, 2000).

En la presente investigación se busca registrar, si existe en el desarrollo educativo del estudiante alguna correlación entre las variables: Nivel educativo del padre /Nivel educativo de la madre y el desarrollo de las subcompetencias lingüísticas de los sujetos, particulamente, la subcompetencia lingüística en L2 (lengua a traducir: inglés) y el de la subcompetencia de transferencia (SCTR). Es decir, establecer si la educación recibida/transmitida por los padres, el ambiente en el que se desarrolla el alumno (con padres universitarios), y el nivel socioeconómico, son factores que influyen en el desarrollo cultural (léase educación), para a partir de ahí, buscar métodos o visualizar estrategias que subsanen las carencias del alumno en su camino hacia la adquisición de la competencia traductora. Implícito en el desarrollo cognitivo del traductor se halla la adquisición, práctica y maduración de la competencia traductora y sus seis subcompetencias, en su camino a la pericia y automatización de la labor.

\section{UBICUIDAD DE LA CULTURA}

De inicio, haremos algunas puntualizaciones. Según la RAE, cultura se define como "conjunto de conocimientos que permite a alguien desarrollar su juicio crítico. Conjunto de modos de vida y costumbres, conocimientos y grado de desarrollo artístico, científico, industrial, en una época, grupo 
social, etc." Por otra parte, para Chinoy (2002, pp. 27-28) en su acepción sociológica, "cultura se refiere a la totalidad de lo que aprenden los individuos como miembros de una sociedad; es un modo de vida, de pensamiento, acción y sentimiento".

La cultura o civilización, tomada en su amplio sentido etnográfico, es ese complejo conjunto que incluye el conocimiento, la creencia, el arte, la moral, la ley, la costumbre y cualquier otra capacidad y hábitos adquiridos por el hombre como miembro de la sociedad (McCurdy, Spradley y Shandy, 2005, p. 5). Lo que nos arroja un todo complejo donde caben todas las habilidades y hábitos del hombre en sociedad.

Por lo tanto, la cultura de una sociedad se basa en la relación mutua que existe entre los mapas mentales individuales y además de crear el imaginario social, es muy

dinámica, pues de acuerdo a Landeros (2016) es muy difícil que una comunidad se mantenga al margen de todos los avances tecnológicos de una cultura global, y que estos no repercutan de una u otra manera en su imaginario y por consiguiente eso provoque cambios que enriquezcan o eliminen su identidad.

\section{CULTURA Y TRADUCCIÓN}

Según Spradley (1972), podemos encontrar tres aspectos de la cultura: conocimientos, comportamientos y artefactos. Luego del establecimiento de que cultura implica saberes declarativos, componentes actitudinales y fabricación y/o manejo de artefactos, definitivamente se puede concluir que las traducciones son productos temporales tanto diacrónicos como sincrónicos. Ya dentro de la traductología, Pym (1992, p. 23) acota que, aunque se cree comúnmente que cuestiones como la definición de cultura son difíciles de construir, más allá del alcance de la teoría de la traducción, su solución podría convertirse en una de las principales aportaciones de los Estudios de Traducción, y asegura que es suficiente con definir los límites de una cultura como los puntos en donde los textos transferidos tienen que ser (intra o interlingüísticamente) traducidos.

Sin embargo, en la actualidad hay gran interés por los estudios interculturales en traducción. Según Olk (2009), durante unos 25 años la competencia intercultural ha sido un concepto muy de moda, citado e influyente en la enseñanza de lenguas. Otro traductólogo, Robinson (1997) establece que "Cultural difference is largely a function of the distance they move, the distance from the place or time in which they are writte $n$ to the place or time in which they are read" (p. 189).

En traductología, la cultura, de acuerdo con Muñoz, "no se contempla como fenómeno interlingüístico ni intercultural, sino interpersonal" (2008, p. 4). Es decir, la traducción como un acto de comunicación entre personas. Definitivamente, la importancia de la cultura es tal, que ahora se habla de 
competencia cultural. Cozma afirma que "the development of cultural competence needs training and this is an aspect that both translator trainers and translation students should have in mind" (2012, p. 67).

En suma, vemos que la cultura compartida dentro y fuera del hogar media el imaginario de los sujetos, sus percepciones y aprendizajes sociales. Por tanto, sus actitudes y comportamientos reproducen la cultura aprehendida y aprendida en sus hogares y ámbitos sociales cercanos.

\section{REFERENTES IDIOSINCRÁSICOS}

Algo particularmente importante es (de ahí la importancia del entorno social y el estatus económico del estudiante) cómo la misma madre reproduce las condiciones de producción (y de la cultura imperante), reforzando inconscientemente el statuo quo, y la ideología coercitiva del Estado, a la manera que el sociólogo Louis Althusser señalaba; para este autor la ideología es ahistórica pues, al igual que el inconsciente freudiano, es eterna; es decir, que siempre habrá ideología. Es decir, el entorno familiar (micro) mantiene y reproduce las condiciones políticas y económicas imperantes establecidas por el Estado con todos los medios (económicos, de comunicación, etc.) a su disposición. Castro-Gómez (2000) lo plantea claramente: "a través de los medios se construyen no solo las grandes ideologías económicas y políticas, sino también ideologías de género, raza, sexualidad y posición social que no son necesariamente reducibles unas a otras" (p.749).

En este tenor, es pertinente acotar que, en la cultura mexicana, y particularmente en las clases medias, la madre es el pilar de la familia, y tradicionalmente es quien se encarga de la educación y la guía de los hijos. Mientras que el rol tradicional del padre es el de proveedor. Aunque, cabe agregar, que en las últimas tres décadas esto ha ido cambiando paulatinamente, según un estudio del año 2014 de la Universidad Autónoma de México (UNAM) publicado en el periódico diario El Universal:

En el México del siglo XXI ya no existe un prototipo de "madre mexicana", al haber diversos modelos de familia. Ahora un creciente número de mujeres se incorpora al mercado laboral y asume la jefatura familiar, cifra que alcanza el 35\% de los hogares nacionales, coinciden Julia Chávez, Gina Zabludovsky y Olga Bustos.

Ahora ya no es posible hablar sólo de la "madre tradicional, la abnegada, que toda la vida la da por los hijos", aseguró al diario Julia Chávez Carapia, una de las participantes de la investigación, y coordinadora del Centro de Estudios sobre la Mujer de la Escuela Nacional de Trabajo Social (ENTS). Sin embargo, no se puede soslayar el papel de las madres como las primeras educadoras en el hogar, situación que confirma lo que aseveran Espejel y Jiménez (2019): en América Latina las madres están presentes en la mayor parte de la educación de los hijos y al tener un nivel de estudio más alto la capacidad para brindar apoyo efectivo a los escolares es mayor. 
Educación, desigualdad y estatus

De inicio, el 'estatus social' lo define Merrian-Webster como el que se basa en a). - Posición o rango en relación con otros. // El estatus de un padre. b). - Rango relativo en una jerarquía de prestigio especialmente: alto prestigio. 2: la condición de una persona o cosa a los ojos de la ley. 3: estado o condición con respecto a las circunstancias el estado de las negociaciones.

Entonces, el estatus difiere de la clase social en que aquel se basa en consideraciones de honor y prestigio, en lugar de la posición puramente económica. En lo relativo a los estudios sobre la correlación entre la raza/etnia y la inequidad educativa en el nivel de secundaria en el Reino Unido, se convirtió en una importante área de estudio desde 1980 en adelante (Stevens, 2007). La desigualdad se ha definido cada vez más en términos de diferencias en los resultados escolares, y el foco de investigación se ha centrado en el papel de la escuela en los procesos que desarrollan tales desigualdades.

Estos hallazgos sugieren una falta de oportunidades para las etnias y razas no blancas en ese país. Sin embargo, no es privativo de esa zona del mundo, Murray, O’Brian y O'Campo (2008) en una crítica a las deficiencias de las actuales reformas educativas en las escuelas de EE.UU., argumentan que, aunque el poder de las escuelas y los educadores para influir en los estudiantes nunca debe ser subestimada, los factores extraescolares asociados con la pobreza desempeñan un papel poderoso y limitante en lo que realmente se puede lograr.

Sobre la educación y desigualdad, Bruner (2004, p. 110) establece que "numerosos grupos e instituciones han subrayado con insistencia que las prácticas educativas y de socialización, tanto anteriores como posteriores al ingreso del niño en la escuela, reflejan y refuerzan las desigualdades propias del sistema de clases".

En el contexto Latinoamericano Espejel y Jiménez (2019), sostienen que varios autores han encontrado que el rendimiento de los alumnos en diferentes niveles académicos tiene relaciones positivas con el nivel socioeconómico de los padres, considerando principalmente la ocupación del padre, debido a que la participación de la mujer en el mercado laboral es baja, a pesar de estar en un punto de inflexión.

En otros hallazgos, las autoras mencionadas muestran que el rendimiento académico de los estudiantes universitarios en México es influenciado principalmente por el nivel de estudios de la madre y la ocupación del padre. Ellas aseguran que su estudio concuerda con otras investigaciones en los diferentes niveles educativos en el mundo. La influencia del nivel de estudios de la madre se debe en gran parte a que es la que participa más activamente en actividades escolares y quien coadyuva al desarrollo de habilidades cognitivas en sus hijos debido a la convivencia, ya que, a pesar del cambio social, son las madres las que se encuentran más relacionadas con la educación de los hijos. 
Si se traslada esta información a nuestro objeto de estudio, vemos que el tratamiento desigual que sufre el estudiante desde el preescolar (kindergarten) hasta el ingreso a los estudios de licenciatura, puede verse reflejado en la disparidad mostrada en los niveles de competencias de los alumnos que han participado en este estudio experimental realizado en la UABC, que inició desde 2006.

Esto muestra que el aprendizaje acumulado por los estudiantes que ingresan a la Facultad de Idiomas, con un promedio de 21 años (Cortez, Basich y Figueroa, 2012, p. 54); además de su cultura adquirida, y su entorno familiar favorable, determinarán su desempeño como estudiantes de traducción, que se pone en práctica a la hora de tomar decisiones y resolver problemas ante un proyecto de trabajo.

Con relación al estatus de los estudiantes de traducción, como se verá más adelante, los sujetos repiten el patrón de sus padres en cuanto a nivel educativo, ya que estos les "modelan" un nivel universitario como forma de vida y estatus en su futuro como profesionistas. Al menos eso es lo que indican los datos.

\section{APRENDIZAJES Y EDUCACIÓN}

Pomerantz, Moorman y Litwack (2007, p. 374) establecen la diferencia entre la educación en casa o la intervención de los padres en la educación de sus hijos, y hacen una amplia distinción entre el involucramiento "based at school and that based at home".

Los autores agregan que los padres en los Estados Unidos, se involucran con más frecuencia en la administración de la escuela a través de su presencia en las juntas generales y pláticas maestros-padres, que según las encuestas nacionales son atendidas por aproximadamente dos tercios de los padres, independientemente de su origen étnico (Departamento de Educación de EE.UU., 2006, en op. cit.).

Además, de acuerdo a la fuente citada, dicha participación es aún mayor entre los padres con estatus socioeconómico alto y elevado nivel educativo. Entre otras puntualizaciones clave, Pomerantz, Moorman y Litwack (2007) destacan que cuando los padres se involucran en la vida académica de los niños con un apoyo a su autonomía, en lugar de ser controladores, los niños pueden beneficiarse en términos de sus logros.

Además, en consonancia con los modelos de desarrollo de motivación, el apoyo autonómico de los padres permite a los niños tomar la iniciativa, lo que les puede llevar a sentir que están a cargo y son capaces de influir en su entorno (Grolnick, Deci y Ryan, 1997). Esto puede aumentar el interés intrínseco en los niños, lo que lleva a comprometerse con su entorno de una manera que mejora el rendimiento.

Por otra parte, a través de los estudios de Hess y Shipman (1965), según lo apunto Bruner (2004), se atrajo la atención hacia el problema de cómo se emplea el lenguaje en la comunicación con niños de corta edad y qué significado tiene para el niño de clase media y baja. Se observó que 
Las madres de clase media solían permitir que sus hijos trabajarán a su ritmo, les hacían sugerencias de carácter estructurador sobre la forma de buscar soluciones a un problema, y les decían que lo que estaban haciendo, lo hacían bien... La estructura general que la madre ofrece al niño le sirve a éste para adquirir disposiciones (estrategias) de aprendizaje que podrían generalizarse a futuras situaciones de resolución de problemas. (Bruner, 2004, p. 117)

Por el contrario, las madres de clase baja no actuaban de manera que el niño se sintiera estimulado a fijarse en las principales características del problema. Sus observaciones eran muy específicas, no resaltaban las estrategias básicas de resolución de problemas y apenas exigían una respuesta por parte del niño. Antes bien, le privaban de la oportunidad de resolver el problema por sí mismo al interrumpir de forma no verbal su actividad de resolución de problemas (Bruner, 2004, p. 118).

Con relación a los aprendizajes Pozo (2005, p. 245) cita a Bandura, quien afirma que "todos nuestros aprendizajes son aprendizajes sociales o culturalmente mediados, en la medida en que se originan en contextos de interacción social, como las relaciones familiares, la escuela, o los ámbitos laborales y profesionales".

Del mismo modo, Pozo establece que la forma más simple de aprendizaje social es posiblemente la adquisición de habilidades sociales, por lo que los primeros modelos a imitar por parte de los hijos, son los padres, de acuerdo a la teoría del aprendizaje de Bandura (1971), quien asegura que a partir de la observación de los demás, uno se forma una concepción de cómo se realizan las nuevas pautas de comportamiento y, en ocasiones posteriores, la construcción simbólica sirve de guía para la acción.

Por su parte, Henson y Eller (2000) reconocen el valor que algunos autores como Zimmerman y Kleefield (1977) y el mismo Bandura (1986) dan al reforzamiento social, y les atribuyen la afirmación de que en el ambiente cotidiano del aula prevalecen los reforzadores sociales entre los que se encuentran el reforzamiento directo, el reforzamiento vicario, y el autorreforzamiento. El reforzamiento directo ocurre durante el proceso de modelamiento. Como se puede establecer, el primer modelamiento proviene del hogar, y son los padres quienes influyen, en primera instancia, sobre la educación y rendimiento escolar de los hijos. Aunque este planteamiento se basa en variables emotivas y abstractas, puede ser determinante en la formación del futuro profesional del sujeto.

\section{MOTIVACIÓN}

En cuanto a la motivación, Pintrich y Schunk (2002) es una fuerza que estimula, sostiene y dirige la conducta hacia una meta. Muchos investigadores encuentran una gran correlacion entre la motivación y el logro (McDermott, Mordell y Stoltzfus, 2001; Weinstein, 1998; en Eggen y Kauchak, 2009). 
Eggen y Kauchak (2009) afirman que los estudiantes bien motivados: procesan la información profundamente y sobresalen en las experiencias de aprendizaje en el aula. Persisten en las tareas difíciles y tienen menos problemas de atención y tienen actitudes más positivas hacia la escuela y ésta les parece satisfactoria. De hecho, en los seres humanos, la motivación intrínseca no es la única forma de motivación, o incluso de actividad volitiva, pero es una que está generalizada y es importante.

Por su parte, Ryan y Deci (2000), explican que estar motivado significa estar movido a hacer algo, y una persona que no siente ímpetus o inspiración a actuar está por tanto desmotivado, mientras que "someone who is energized or activated toward an end is considered motivated" (p. 54).

La motivación intrínseca se define como la realización de una actividad por sus satisfacciones inherentes más que por algunas consecuencias distintas. Cuando una persona intrínsecamente motivada es movida a actuar por diversión o el desafío que conlleva, más que por la causa externa, beneficio o recompensa (Ryan y Deci, 2000).

Lefton y Brannon (2006) afirman que uno de los enfoques más famosos y utilizados para la comprensión de las motivaciones es la jerarquía de necesidades de Abraham Maslow. Maslow, agregan los autores, entendió la distinción entre motivaciones primarias y secundarias, y amplió este concepto para incluir una secuencia y priorización de estas motivaciones, donde en la punta de la pirámide, la autorrealización, sugiere el alcance de todo el potencial relacionado con la creatividad y productividad.

En este tenor, los psicólogos Wright y Wiedeger (2007) estudiaron cómo los comportamientos motivados se inician de acuerdo a las necesidades: de cómo el estado de necesidad se vuelve una meta a conseguir, y una vez alcanzada, cómo un sistema de recompensas refuerza la secuencia de comportamientos exitosos. Esta solución del comportamiento se almacena en la memoria a largo plazo para que así pueda ser recuperada más tarde en circunstancias similares, por lo que provee ventajas al comportamiento futuro del individuo. Haciendo un símil, estas estrategias las viven los traductores a lo largo de su desempeño profesional en todos los aspectos relacionados con el proceso de entrega de un proyecto de traducción.

Ante esto, nuestra meta como facilitadores es elevar al máximo la motivación intrínseca por aprender de nuestros alumnos de traducción, por lo cual planteamos desde hace tiempo que: "Situar el aprendizaje y rodear a los estudiantes de ambientes más realistas los provee de aprendizajes significativos y de mayores destrezas como aspirantes al conocimiento experto en traducción" (Cortez, 2009, p. 290).

\section{MATERIALES Y MÉTODOS}

En nuestro estudio se analizaron y midieron seis variables, integrantes del modelo de la competencia traductora de PACTE (2000, 2002, 2003, 2005, 2008, 2009, 2011, 2014, 2019) que se ponen 
en juego durante la realización de cada proyecto de traducción. Estas competencias se encuentran integradas en el plan de estudios del grado (2006 y 2017) de la UABC.

Las variables analizadas fueron la subcompetencia lingüística en L1 y L2, subcompetencia de transferencia (¿el estudiante sabe traducir?), subcompetencia profesional/instrumental, subcompetencia contrastiva, psicofisiológica y terminológica. De estas variables integradas en una de tres matrices, se seleccionaron los resultados de la medición de la subcompetencia de transferencia (que es la esencia de la Competencia Traductora; CT), la subcompetencia lingüística en L2, que implica el manejo de la lengua a traducir (inglés), y que registramos con un examen Pre-Toefl (1991) para contrastarlas con un estudio socioeconómico aplicado a toda la muestra (Cortez, 2014).

Los datos fueron almacenados en tres matrices que nombramos (Ver Tabla 1, infra):

1. Matriz del Estudio socioeconómico (ESOC).

2. Matriz de los Cuestionarios I (entrada pre-tarea) y II (salida, pos-tarea)

3. Matriz del Cruzamiento de Variables 11 (ingreso mensual de los padres), 12 (Estudios de la madre), 13 (Estudios del padre), 21 (Subcompetencia de Tranferencia) y 22 (Subcompetencia Lingúística en L2; inglés).

Basados en un estudio de la Asociación Mexicana de Agencias de Investigación de Mercado y Opinión Pública A.C (2006) establecimos tres estratos para ubicar a los sujetos: Escogimos tres categorías:

1. Mayor de 20 mil pesos mexicanos o Estrato 1 (E1), o ingreso mayor a 1,509 dólares estadounidenses.

2. Ingresos de entre 10 y $20 \mathrm{mil}$ pesos (equivalentes a 1,509 dólares estadounidenses) o Estrato 2 (E2),

3. Ingresos mensuales de los padres hasta 10 mil pesos (equivalentes a 754 dólares estadounidenses al tipo de cambio de 13.25 pesos por dólar) o Estrato 3 (E3).

La traducción se capturó dentro del programa Translog2000user, y convertida a .rtf en la paquetería Word 2010 de Microsoft para su revisión en Translog2000.

Como parte del análisis triangulado del producto-proceso (observación participante, cuestionario de entrada y salida, captura de la traducción en Translog2000, examen Pre-Toefl y la ponderación de la subcompetencia de Transferencia) se buscó la posible correlación entre la calidad de las traducciones y el rango de la subcompetencia de Transferencia reflejada en los productos calificados por profesores de la Facultad. Esta variable en sí es el reflejo sincrónico del alumno para utilizar su subcompetencia de 
translación de L1 a L2, la intención era detectar si podían o no realizar ese encargo de traducción, atendiendo el nivel intermedio del grado que cursaban.

También se hizo énfasis en el uso del Protocolo Previo de Traducción (PPT) (Cortez, 2009, 2014, 2018, 2019, 2019b), que es una metodología adaptada del Modelo de Competencia Traductora de PACTE (2003), en un afán de registrar su utilidad como estrategia, y a manera de la búsqueda de nuevas metodologías en la enseñanza de la traducción, y de andamiajes prácticos que ayuden a mejorarla. Somos conscientes de que la aplicación del PPT resulta una estrategia artificial, pues los profesionales de la traducción utilizan el método que mejor consideran a la hora de trabajar (nosotros mismos lo vivimos). El PPT fue una estrategia tanto de investigación, como de apoyo a la metacognición de los estudiantes, para que fueran conscientes de los pasos que se integrarán en un futuro a su "chip traductor". La finalidad fue poner el proceso y observarlo sobre la placa de Petri de manera más aséptica posible.

Translog2000, versión 1.0 Beta (4) es un programa desarrollado por Arnt Lykke Jakobsen y Lasse Schou para el sistema operativo Windows (De Rooze, 2008, p. 18) y fue creado como alternativa a la recogida de datos mediante protocolos de pensamiento en voz alta, y permite guardar y estudiar toda captura de texto (key-logging) sobre un teclado de computadora dentro del entorno del programa. Translog2000 registra la información del tiempo exacto en el que se realiza cada tecleado y permite reproducir en la pantalla del ordenador el proceso de captura.

El baremo utilizado por los correctores/revisores fue de 0 a 100 y registra si el alumno sabe traducir; es decir, tiene desarrollada, lo que Hurtado (1996) denomina, la competencia de transferencia. También se utilizó el mismo para medir el rendimiento de los estudiantes en el Pre-Toefl. Esta escala de calificación se encuentra regulada en el artículo 65 del Estatuto Escolar de la UABC.

Es importante aclarar que, ante la falta de estudios relacionados con el tema en 2006, establecimos nuestro propio baremo para registrar el equilibrio/desequilibrio entre la CT y el resultado del Pre-Toefl (PT). Pensamos que la diferencia no debe ser mayor a 15/100 puntos porcentuales entre una y otra. Así, tenemos que si el sujeto alcanza 100/100 es excelente en la variable medida, 90-99/100 es una calificación muy buena, y 80-89/100 es buena, 70-79/100 regular y 60-69/100, suficiente a pobre y 59/100 o inferior, insuficiente o no aprobado. Por lo que, si un sujeto presenta una CT de 90/100 y un PT de 60/100, nos dice que hay un desbalance en la adquisición de una y otra.

Con el fin de alcanzar un margen de error de 7\%, con un nivel de confianza de 95\%, nuestro universo es de 125 sujetos con un nivel de heterogeneidad de 50\%, por lo que el tamaño muestral recomendado es de 77 individuos (Netquest, 2020). Nuestra muestra constó, de 81 alumnos, por tanto, los resultados se pueden generalizar a nuestro ámbito, que es Mexicali. 
El texto utilizado durante este estudio, seleccionado para realizar una traducción del inglés al español, fue de 412 palabras con el tema de miopía del tipo informativo- científico y fue tomado de Internet. Una vez convertido a extensión tpl para su traducción dentro del programa Translog2000user por los estudiantes, se envió para su posterior calificación a cuatro profesores con cinco años de experiencia en la enseñanza de traducción.

\section{RESULTADOS Y DISCUSIÓN}

En principio, caracterizaremos algunos de los datos obtenidos en el ESOC de nuestro objeto de estudio: uno de ellos es que a lo largo de este estudio longitudinal (2006-2013) hemos sido testigos de la deserción y abandono de los estudios por parte de los alumnos de licenciatura, los cuales, una vez que cursan y terminan el tercer semestre, es más probable que egresen. De ahí la importancia de buscar variables que los motiven en su desarrollo y adquisición de la competencia traductora. Por lo tanto, es importante saber si las variables estudiadas tienen alguna incidencia en el desarrollo de las competencias y el logro académico.

Figura 1. Como puede apreciarse en la gráfica, en nueve semestres, sólo el $27.16 \%$ de los estudiantes de quinto semestre son varones.

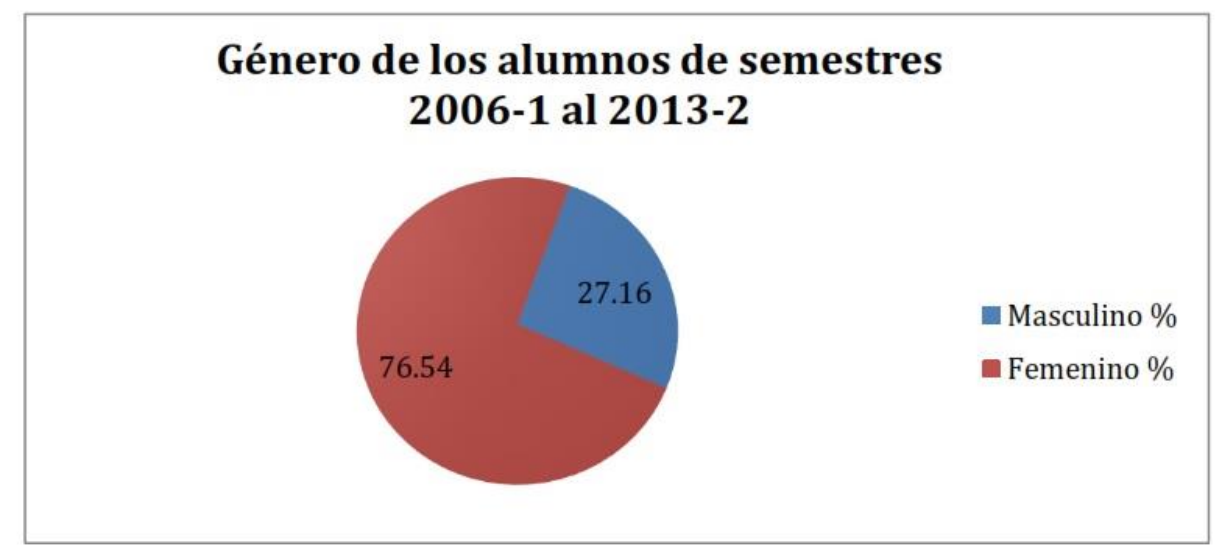

Fuente: Cortez (2014).

En la muestra vemos que el $76.54 \%$ de los estudiantes son mujeres y $27.16 \%$ varones (Figura 1); lo que representa que por cada hombre que egrese, habría tres egresadas que podrán dedicarse también a la traducción e interpretación. 
Figura 2. Existe una preeminencia (42\%) de sujetos con madres egresadas de estudios superiores. Fuente: Cortez (2014).

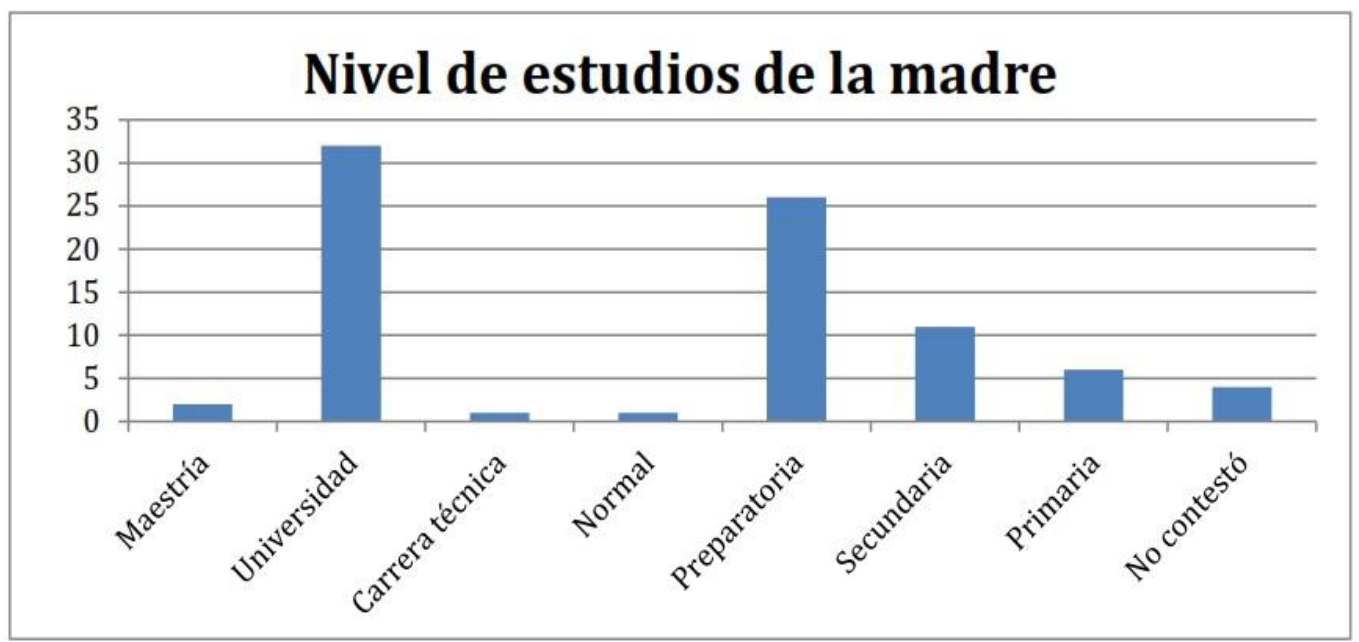

De acuerdo con la Figura 2, el nivel educativo dominante en las madres de los sujetos de la muestra es el de universidad, seguido de los estudios de preparatoria. Esta tendencia favorece el modelaje de una madre educada, profesionista, y con una mayor cultura. El $42 \%$ de los sujetos de la muestra dijo tener una madre con estudios universitarios. Cabe mencionar que la maestría es el grado de estudios máximo entre las madres de los sujetos.

Figura 3. También los padres con estudios universitarios de los sujetos estudiados forman mayoría, 54.32\%, frente a los otros niveles de la muestra. Fuente: Cortez (2014).

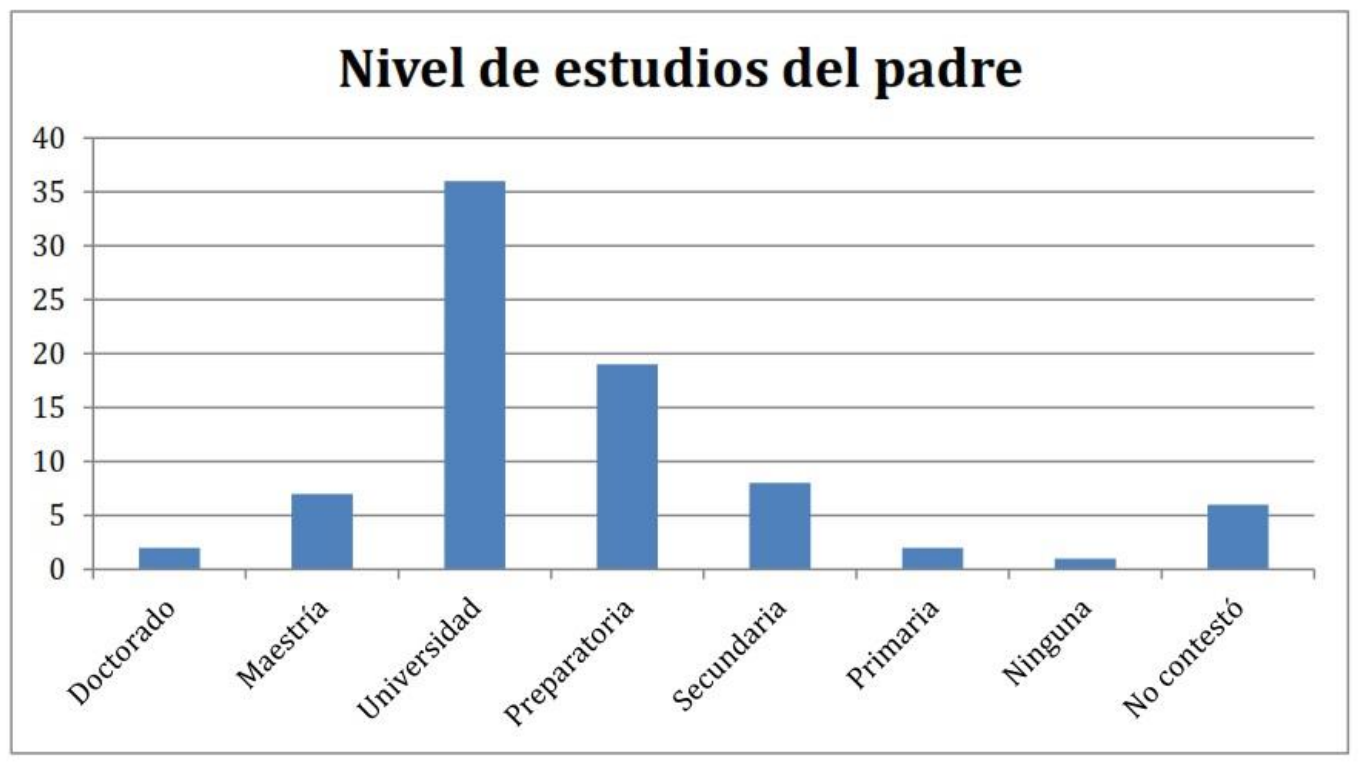

En cuanto a los padres de los sujetos (Figura 3), aparece el doctorado como límite educativo superior, prevaleciendo aquellos con estudios universitarios, seguidos de los egresados de preparatoria, lo 
que en sí es una tendencia favorable para el desarrollo futuro de los estudiantes. Es destacable que el $54.32 \%$ de la muestra externó que su padre había cursado estudios universitarios.

Como un ejemplo claro de motivación intrínseca podemos mencionar al sujeto 16 (Tabla 1, infra), quien a pesar de contar con una madre con sólo estudios de primaria y un padre sin alfabetización formal, alcanzó una SCTR de 85,66/100 y un SLL2 de 71/100; esto sería la otra cara de la moneda, una extraordinaria motivación intrínseca y un deseo de avanzar en la escala social por medio de la educación.

\section{A MAYOR EDUCACIÓN EN PADRES, MAYOR SCTR Y SLL2}

En este apartado analizamos sólo al Estrato 3 y los sujetos cuya puntuación en la SCTR es de 80/100 y mayor (Tabla 1). Buscamos alguna relación, a partir de los datos, entre la educación de sus padres y el rendimiento escolar de los sujetos.

Tabla 1. En la Matriz de la Subcompetencia de Transferencia buena y muy buena del Estrato 3.

\begin{tabular}{|c|c|c|c|c|c|}
\hline SUJETOSDEE3 & VAR. II & VAR. 12 & VAR. 13 & VAR.2I & VAR.22 \\
\hline $\begin{array}{l}\text { SEMESTRES } \\
2006-2013\end{array}$ & $\begin{array}{l}\text { INGRESO MENSUAI. } \\
\text { DE LOS PADRES }\end{array}$ & $\begin{array}{l}\text { NIVEI ESTUDIOS- } \\
\text { MADRE }\end{array}$ & $\begin{array}{l}\text { NIVEL ESTUDIOS- } \\
\text { PADRE }\end{array}$ & $\begin{array}{c}\text { SUBCOMPETENCIA } \\
\text { DE } \\
\text { TRANSFERENCIA }\end{array}$ & PRE-TOEFL \\
\hline ALUMNO1 & $10 \mathrm{MIL}$ & PREPARATORIA & UNIVERSIDAD & 95 & 64 \\
\hline ALUMNA 2 & $10 \mathrm{MIL}$ & SECUNDARIA & UNIVERSIDAD & 93.33 & 62 \\
\hline ALUMNA 3 & $10 \mathrm{MIL}$ & SECUNDARIA & PREPARATORIA & 93 & 72 \\
\hline ALUMNA 4 & $10 \mathrm{MIL}$ & CARRERA TEECNICA & SECUNDARIA & 92.33 & 63 \\
\hline ALUMNA 5 & $10 \mathrm{MIL}$ & UNIVERSIDAD & PREPARATORIA & 91.66 & 80 \\
\hline ALUMNO 6 & $10 \mathrm{MIL}$ & UNIVERSIDAD & UNIVERSIDAD & 91 & 651 \\
\hline $\begin{array}{c}\text { AIUMNA } 7 \\
\text { (MÊRITO ESCOLAR) } \\
\end{array}$ & $10 \mathrm{MIL}$ & UNIVERSIDAD & UNIVERSIDAD & 90 & 83 \\
\hline ALUMNA 8 & $10 \mathrm{MIL}$ & UNIVERSIDAD & PREPARATORIA & 75 & 83 \\
\hline $\begin{array}{c}\text { AIUMNA } \\
\text { (MERTIO ESCOLAR) }\end{array}$ & $10 \mathrm{MIL}$ & PREPARATORIA & SECUNDARIA & 90 & 77 \\
\hline ALUMNA 10 & $10 \mathrm{MIL}$ & PREPARATORIA & PREPARATORIA & 90 & 73 \\
\hline ALUMNA II & $10 \mathrm{MIL}$ & PRIMARIA & PREPARATORIA & 90 & 72 \\
\hline ALUMNA 12 & $10 \mathrm{MIL}$ & SECUNDARIA & SECUNDARIA & 90 & 61 \\
\hline ALUMNA 13 & $10 \mathrm{MIL}$ & PRIMARIA & PRIMARIA & 89.33 & 55 \\
\hline ALUMNO14 & $10 \mathrm{MIL}$ & PREPARATORIA & SECUNDARIA & 88 & NO/CONT. \\
\hline ALUMNA 15 & $10 \mathrm{MIL}$ & PREPARATORIA & UNIVERSIDAD & 87.66 & 78 \\
\hline ALUMNO 16 & $10 \mathrm{MIL}$ & SECUNDARIA & $\mathrm{NO} / \mathrm{CONT}$ & 87.66 & 73 \\
\hline ALUMNO17 & $10 \mathrm{MIL}$ & UNIVERSIDAD & PREPARATORIA & 87.33 & 90 \\
\hline ALUMNO 18 & $10 \mathrm{MIL}$ & UNIVERSIDAD & PREPARATORIA & 86.25 & 58 \\
\hline ALUMNA 19 & $10 \mathrm{MII}$ & PREPARATORIA & UNIVERSIDAD & 86 & 67 \\
\hline ALUMNA 20 & $10 \mathrm{MIL}$ & UNIVERSIDAD & PREPARATORIA & 85.75 & 79 \\
\hline ALUMNA 21 & $10 \mathrm{MIL}$ & PRIMARIA & SIN ESTUDIOS & 85.66 & 71 \\
\hline ALUMNO 22 & $10 \mathrm{MIL}$ & MAESTRIA & PREPARATORIA & 85 & 82 \\
\hline ALUMNO 23 & $10 \mathrm{MIL}$ & $\begin{array}{l}\text { ESCUILA NORMAI } \\
\text { FRONIERIZA PARA } \\
\text { MAESTROS }\end{array}$ & PREPARATORIA & 85 & 80 \\
\hline ALUMNA 23 & NO/CONT. & UNIVERSIDAD & UNIVERSIDAD & 82.75 & 69 \\
\hline ALUMNA 25 & $10 \mathrm{MIL}$ & UNIVERSIDAD & PREPARATORIA & 82.50 & 65 \\
\hline ALUMNA 26 & $10 \mathrm{MIL}$ & PREPARATORIA & UNIVERSIDAD & 80 & 82 \\
\hline ALUMNA 27 & $10 \mathrm{MIL}$ & PRIMARIA & UNIVERSIDAD & 85 & 58 \\
\hline ALUMNA 28 & $10 \mathrm{MIL}$ & PRIMARIA & PRIMARIA & 80 & 69 \\
\hline ALUMNA 29 & $10 \mathrm{MIL}$ & UNIVERSIDAD & UNIVERSIDAD & 80 & 62 \\
\hline AI.UMNA 30 & $10 \mathrm{MIL}$ & SECUNDARIA & UNIVERSIDAD & 80 & 53 \\
\hline
\end{tabular}

Fuente: Cortez (2014). 
La Tabla 1 indica que el 38,46\% de la muestra, ubicado en el E3, tiene una SCTR de buena a muy buena; y, además, en este sector se ubican dos premios al Mérito Escolar; es decir, dos alumnos fueron reconocidos por la Universidad por haber obtenido el más alto promedio de calificación de su generación. Algo particular, es que el 60\% de los sujetos se encuentra dentro de los promedios de 80/100 hacia arriba, donde la variable "padre universitario" se halla presente.

Con el fin de interpretar los datos, tomamos como referente el nivel de estudio más alto alcanzado, ya sea por el padre o la madre, de los sujetos del E3 incluidos en la Tabla,

en la cual se concentran los sujetos del E3 con una CT de 80/100 y mayor de todos los semestres analizados en este estudio. Tras el análisis de los datos arrojados por el estudio, podemos establecer los siguientes hallazgos:

1. Dentro de los diez primeros lugares de SCTR en el sector del E3, el $20 \%$ manifestó tener al menos un progenitor con estudios universitarios, es decir, en esos hogares se tiene el "conocimiento del mundo" y la visión de un universitario. Además, eso se repite en el 55,17\% de todo este sector del E3.

2. En los diez primeros lugares, el segundo nivel educativo que predomina en los padres de los sujetos es el de preparatoria. En el sector, la preparatoria ocupa un 17,24\%. Sólo un sujeto cuya madre estudió una carrera técnica ocupa el lugar número 4 en la tabla.

3. El tercer nivel educativo que predomina en los hogares del sector es la primaria, ya sea en uno o en ambos padres, con un 10,34\%. Aquí cabe destacar la fuerte motivación de los sujetos 13, 21 y 28 quienes, a pesar de no contar con padres en niveles superiores de educación, presentan SCTR de 80 a 89.33/100, y una SLL2 de 69 a 90/100. Ellos son la excepción a la regla, y su deseo por destacar los ha llevado a esas puntuaciones, a pesar de una serie de variables en su contra, como la misma economía.

4. Algo a destacarse es que en el grupo con la variable "padre universitario" tenemos calificaciones ligeramente mayores en SLL2 con una media de 71.11 y en SCTR: 85.79 como media. Mientras que el otro sector obtuvo una media de 63.83 en SLL2 y algo, muy positivo: 88.41 de media en SCTR. Esto lo concuerda con un deseo a destacar y su expectativa de subir en la escala social. Cabe aclarar que tres sujetos no aprobaron la SLL2 en el primer sector mencionado y uno en el segundo.

Sobre el factor económico, un estudio chileno sobre la escuela pública básica de Sánchez, Reyes y Villarroel (2016) ubica las expectativas de los padres como una variable mediadora entre el nivel socioeconómico y el logro académico, donde "los padres que tienen altas expectativas educativas para sus hijos, independientemente de la posición social, son más propensos desarrollar en los niños altas expectativas educativas para sí mismos". Por tanto, las personas de nivel socioeconómico bajo que han 
aumentado las expectativas educativas son más propensas a tener expectativas laborales similares a las de sus pares de nivel socioeconómico más alto (Schmitt-Wilson, 2013).

5. En el cuarto nivel de educación encontramos la secundaria con 6,89\%.

6. En un 3,44\% ubicamos la maestría, y la carrera técnica antes mencionada, lo que sumados, da un total de $6,88 \%$.

7. Además de los dos sujetos (7 y 9) que obtuvieron el Mérito Escolar (con padres que estudiaron universidad y preparatoria como referentes) dentro del E3, podemos destacar los logros de los sujetos 4, $12,13,16,21$, y 28 , quienes, a pesar de que los niveles educativos de sus padres van de cero a carrera técnica, comprendieron que la herramienta de movilidad social por tradición ha sido la educación. Esto les proporcionará mayores oportunidades de desarrollo profesional en su futuro. En resumen, del sector E3 con mejores calificaciones, el 30\% no presentó la variable padre universitario, contra el 70\% que sí.

Otro dato a destacar es que cuatro de los primeros diez sujetos aparecen con una madre que cursó estudios universitarios, lo que coincide con la oportunidad creciente de acceso de las mujeres a la educación superior que hemos estado percibiendo en las matrices elaboradas, donde por cada egresado varón en traducción, habrá tres mujeres que se dedicarán a la misma profesión, ese patrón se sostiene en este estudio (Cortez, 2014,

2018). Este fenómeno lo confirma también ANUIES $(1970,1991)$ que reporta la participación femenina en la educación superior pasó de ser sólo de 15\% en 1970 a $40 \%$ al comienzo de los años noventa. En la Tabla 2 se puede apreciar la lista de los primeros cinco mejores promedios de cada estrato económico desde 2006 a 2013, donde se registra la presencia de la variable "padre universitario" y su influencia (entre otros factores) en las puntuaciones obtenidas en la Subcompetencia de Transferencia por parte de los estudiantes de traducción. La educación paterna es un modelo a seguir y una motivación externa positiva en su futuro desarrollo profesional.

Vemos que en los estratos económicos donde el nivel es superior a los 10 mil pesos mensuales (E1 y E2), el nivel de estudios de los padres es de universitarios o superior. Lo que abona a nuestra hipótesis.

Aunque el corte en los resultados del presente estudio es del 2013, el estudio de Espejel y Jiménez (2019) concluye con resultados similares: ellas encontraron que el rendimiento académico de los estudiantes universitarios es influenciado principalmente por el nivel de estudios de la madre y la ocupación del padre; y a su vez concuerdan con los hallazgos de diversos investigadores en los diferentes niveles educativos en el mundo. 
Tabla 2. Presencia de la variable "Padre universitario“ en los cinco primeros lugares de Subcompetencia de Transferencia de toda la muestra.

\begin{tabular}{|c|c|c|c|c|c|c|}
\hline \multicolumn{7}{|c|}{ Variable "padre universitario" y puntajes de SCTR en la muestra } \\
\hline \multirow[t]{2}{*}{ Semestres } & \multirow{2}{*}{$\begin{array}{l}\text { Porcentaje del } \\
\text { estrato con } \\
\text { padre/madre } \\
\text { universitario/a }\end{array}$} & \multirow{2}{*}{$\begin{array}{c}\text { Puntuaciones en } \\
\text { Subcompetencia de } \\
\text { Transferencia dentro } \\
\text { de los primeros cinco } \\
\text { lugares }\end{array}$} & \multirow{2}{*}{$\begin{array}{l}\text { *Pertenencia } \\
\text { a ESOC de los } \\
\text { stujetos }\end{array}$} & \multicolumn{3}{|c|}{$\begin{array}{l}\text { Aqui se ubican los sujetos que } \\
\text { manifestaron no tener padres } \\
\text { universitarios y que se ubican en los } \\
\text { primeros cinco lugares de SCTR. }\end{array}$} \\
\hline & & & & Otros niveles & Puntuación & ESOC \\
\hline $\begin{array}{l}2006-1 \\
2006-2 \\
2007-1 \\
2007-2 \\
\end{array}$ & $50 \%$ & $100 / 100 / 100 / 80$ & $\begin{array}{l}\mathrm{E} 1: 2 \\
\mathrm{E} 2: 2\end{array}$ & Preparatoria & 88 & E3:1 \\
\hline $2008-2$ & $77.77 \%$ & $95 / 90 / 90 / 85$ & $\begin{array}{l}\mathrm{E} 1: 2 \\
\mathrm{E} 3: 3 \\
\end{array}$ & Secundaria & 90 & $E 3: 1$ \\
\hline $2009 \cdot 2$ & $92.30 \%$ & $99 / 99 / 80 / 80 / 80$ & $\begin{array}{l}E 2: 2 \\
E 3: 3\end{array}$ & & & \\
\hline $2011-2$ & $80 \%$ & $93 / 93 / 91 / 90 / 90$ & $\begin{array}{l}\mathrm{E} 1: 3 \\
\mathrm{E} 2: 1 \\
\mathrm{E} 3: 1\end{array}$ & & & \\
\hline 2012.2 & $72.72 \%$ & $\begin{array}{c}89.75 / 86.25 / 86.25 \\
85.75\end{array}$ & $\begin{array}{l}\mathrm{E} 2: 2 \\
\mathrm{E} 3: 2\end{array}$ & Preparatoria & 83.75 & $E 3: 1$ \\
\hline 2013.2 & $47.36 \%$ & $93.33 / 92 / 92$ & $\begin{array}{l}E 2: 1 \\
E 3: 2\end{array}$ & $\begin{array}{c}\text { Preparatoria/ } \\
\text { Carrera } \\
\text { Tecnica } \\
\end{array}$ & $93 / 9233$ & $\begin{array}{l}E 3: 1 \\
E 2: 1\end{array}$ \\
\hline Totales & $\begin{array}{l}70.02 \% \text { de } \\
\text { promedio. }\end{array}$ & $\begin{array}{c}\text { Promedio } \\
90.41\end{array}$ & $\begin{array}{l}\mathrm{E} 1: 7 \\
\mathrm{E} 2: 8 \\
\mathrm{E} 3: 11\end{array}$ & $\begin{array}{l}\text { Preparatoria, } \\
\text { mayor nivel. }\end{array}$ & $\begin{array}{c}\text { Promedio } \\
89.41\end{array}$ & $\begin{array}{l}\mathrm{E} 2: 1 \\
\mathrm{E} 3: 4\end{array}$ \\
\hline
\end{tabular}

Claves: * Se tomaron los cuatro semestres como un estrato por su captura en SPSS, con una muestra de 18 sujetos de un total de 39. ** E1: Ingresos mayores a 20 mil pesos mensuales. E2: Hasta 20 mil pesos. E3: Hasta 10 mil pesos. Fuente: Cortez (2014).

La influencia del nivel de estudios de la madre se debe en gran parte a que es la que participa más activamente en actividades escolares y quien coadyuva al desarrollo de habilidades cognitivas en sus hijos debido a la convivencia, ya que, a pesar del cambio social, son las madres las que se encuentran más relacionadas con la educación de los hijos.

En la misma línea, la Tabla 3 muestra los seis mejores lugares de calificación en la SLL2 (PreToefl) del E3, con mayor puntuación en la SCTR, y con un mejor balance entre las dos variables medidas, muestra la presencia de la variable "padre/madre universitario/a", o incluso un mayor nivel.

Tabla 3. Preeminencia de la variable padre universitario en los mejores promedios en SLL2 que van de 80 a 90/100 en el E3.

\begin{tabular}{|c|c|c|c|c|c|c|}
\hline & $\begin{array}{l}\text { SUTETOS } \\
\text { DELE3 }\end{array}$ & $\begin{array}{l}\text { VAlर II } \\
\text { ENGAFSO }\end{array}$ & $\begin{array}{l}\text { VRRT2 } \\
\text { NIVHE ESTUDIOS- } \\
\text { MADRE }\end{array}$ & $\begin{array}{l}\text { VARTB } \\
\text { NIVIL ESTUDEOS } \\
\text { PADRE }\end{array}$ & $\begin{array}{l}\text { VRK 2 } \\
\text { Scik }\end{array}$ & $\begin{array}{l}\text { VRR } 22 \\
\text { S.1.2 }\end{array}$ \\
\hline 1 & ALUMNOH & $10 \mathrm{MII}$ & UNIVERSADAD & PREPARATORLA & 87.33 & 90 \\
\hline 2 & $\begin{array}{l}\text { AIUMNA } 4 \\
\text { (MFRITO FSCOLAR) }\end{array}$ & to MII. & UNIVERSIDAD & UNIVERSIDAD & 90 & 83 \\
\hline 3 & ALMNAS & $10 \mathrm{MII}$. & UNIVERSADAD & PREPARATORLA & 90 & 83 \\
\hline 4 & AuMvo is & $10 \mathrm{MIL}$ & MAESTRLA & PREPARATORLA & 85 & 82 \\
\hline 3 & AHUMNA 21 & $10 \mathrm{MII}$ & PRFPARATORLA & UNINERSIDAD & 80 & 82 \\
\hline 6 & AUMNA 5 & to MII. & UNTVERSIDAD & DREDARATORLA & 91.66 & 80 \\
\hline
\end{tabular}

Fuente: Cortez (2014). 
En cuanto a la variable 22 (Tabla 3), y su relación con la 12 y 13, podemos mencionar que:

1. Las variables 11 y 12 que encajan con el factor "padre universitario" están presentes en la tabla de los "Mejores promedios en SLL2 en E3", pues 5 de los seis sujetos manifestaron tener ese estatus, y en el caso restante, la madre cuenta con nivel de maestría. El otro sector del E3 quedó por debajo de 80/100 en la SLL2. En una investigación sobre estudios preuniversitarios en EU (College) Moon y Bouchey (2019, p. 465) afirman que "Students of college educated parents who perceived that their parents placed a higher value on a college education were more likely to think finishing college is important", lo cual puede verse como modelo a seguir, de igual modo.

2. En este análisis vimos aún más clara la relación entre el rendimiento escolar y la importancia de la educación de los padres en el futuro desarrollo de los sujetos. Sólo dos sujetos varones entraron en la tabla, la mayoría fueron mujeres.

\section{CONCLUSIONES}

Por supuesto que la realidad no es lineal, pero lo que hemos percibido hasta ahora es un reflejo de cómo una educación ascendente en los padres puede modelar o servir de ejemplo en el aprendizaje social de los hijos, además de motivarlos a que suban en la escala social por medio de la educación. Esta correlación se explica por la presencia de la variable padre/madre universitario/a y de cómo el nivel educativo de los padres sí influye positivamente como motivación extrínseca en los hijos, modelando sus deseos de autorrealizarse, alcanzando el estatus educativo y económico de sus progenitores.

Durante el análisis de los datos obtenidos de los sujetos ubicados en el E3, hemos deducido, a partir de los datos, la existencia de variables motivacionales que emergen de los distintos estratos como:

a) Estudiar para ascender en la escala social. b) Cursar una carrera universitaria.

c) Alcanzar o superar el nivel de desarrollo/estatus de los padres. d) Los resultados, como en otros estudios similares, permitieron comprobar que a nivel universitario, el rendimiento académico de los estudiantes es influenciado por la variable padre universitario, y que aquellos que tienen ocupaciones con mejores ingresos (E1 y E2) pueden favorecen el uso de recursos para el aprendizaje de una segunda lengua (lengua a traducir) y mejorar sus competencia traductora holística, como lo muestran sus puntajes alcanzados.

En el E3, menos favorecido económicamente, se percibe una fuerte motivación intrínseca por sobresalir en la escala social, y nuevamente, vemos presente en los puntajes más elevados de Subcompetencia de Transferencia y Subcompetencia Lingüística en L2, la presencia del factor "padre universitario", por lo que la lógica nos lleva a deducir que existe una correlación en los resultados. 
Sin embargo, la medición exacta del fenómeno y el grado de la motivación como variable independiente nos llevaría al campo de la psicología de la motivación, con lo que se requieren baremos específicos y una entrevista estructurada personal con cada sujeto, para conocer de viva voz las motivaciones más profundas, lo cual implica una investigación integral en sí misma. 


\section{REFERENCIAS}

ANUIES (1970). Anuario estadístico, México: ANUIES. ANUIES (1991). Anuario estadístico, México: ANUIES.

Althusser, L. (1988). Ideología y aparatos ideológicos del estado. Freud y Lacan, Buenos Aires: Nueva Visión.

Bandura, A. (1987). Teoría del aprendizaje social. España: Espasa Universitaria.

Bandura, A. (1971). Psychological modelling: Conflicting theories. Chicago: Aldine-Atherton.

Basich, K. (2012). La formación de profesores de traducción. Reflexiones desde un caso mexicano. Mexicali: Universidad Autónoma de Baja California.

Bruner J. (2004). Desarrollo Cognitivo y Educación. Selección de textos por Jesús Palacios.

Madrid: Morata.

Castro-Gómez, S. (2000). Althusser, los estudios culturales y el concepto de ideología.

Revista Iberoamericana, 66 (193), octubre-diciembre 2000, 737-751.

Chinoy, E. (2002). Introducción a la Sociología. Conceptos básicos y aplicaciones. México, D.F.: Paidós.

Cortez, J. (2019). socio-economic level and its influence on the acquisition of translation competence. Sendebar, 30, 357-388. http://dx.doi.org/10.30827/sendebar.v30i0.6594

Cortez J. (2019b). Registro y Evolución de la Subcompetencia Extralingüística en estudiantes de traducción. Onomázein, 4 (46). DOI:10.7764/onomazein.46.10.

Cortez, J. (2018). La formación de competencias en traducción: Un caso mexicano, en CIEBC 2018 (Ed.), XIV Congreso Internacional sobre el Enfoque Basado en Competencias: Aprendizaje, Formación y Educación por Competencias, (pp. 283-302). Editorial Corporación CIMTED. https://bit.ly/34jXs8T

Cortez, J. (2014). El nivel socioeconómico de los estudiantes de traducción en la Facultad de IdiomasMexicali de la UABC, como variable condicionante en la adquisición de la competencia traductora: Un estudio exploratorio [tesis doctoral, Universidad de Granada]. Repositorio de la UGR. https://bit.ly/34lvVUy

Cortez, J., Basich, K. y Sánchez, I. (2012, agosto). La subcompetencia instrumental/profesional en la formación de traductores. Trabajo presentado en el V Congreso Internacional de Traducción e Interpretación. Traduciendo Culturas: Más que un desafío. Mexicali. https://bit.ly/31e8tq4

Cortez, J. (2009). Nuevas metodologías en enseñanza de la traducción: Una perspectiva cognitiva. Mutatis Mutandis, 2 (2), 282-294.

Cozma, M. (2012). Perceptions of Cultural Competence: the trainees' perspective.

Professional communication and translation studies, 5 (1-2), 67-74.

Cultura. (2021). En Diccionario de la Lengua Española (en línea). Recuperado de https://dle.rae.es/cultura?m=form 
De Rooze, B. (2008). La Traducción contra Reloj. En M. Fernández y R. Muñoz Martín (Eds.), Aproximaciones Cognitivas al Estudio de la Traducción y la Interpretación (pp. 1-28). Comares: Granada.

Eggen, D y Kauchak, D. (2009). Estrategias Docentes. Enseñanza de contenidos curriculares y desarrollo de habilidades de pensamiento. México, D.F.: Fondo de Cultura Económica.

Espejel, M. V. y Jiménez, M. (2019). Nivel educativo y ocupación de los padres: Su influencia en el rendimiento académico de estudiantes universitarios. RIDE (Revista Iberoamericana para la $\begin{array}{lllllll}\text { investigación y el desarrollo educativo), } 10 & \text { (19), Julio- Diciembre. }\end{array}$ https://doi.org/10.23913/ride.v10i19.540

Grolnick, W. S., Deci, E. L., \& Ryan, R. M. (1997). Internalization within the family:

The self-determination theory perspective. En J. E. Grusec \& L. Kuczynski (Eds.),

Parenting and children's internalization of values: A handbook of contemporary theory (pp. 135-161). John Wiley \& Sons Inc

Henson, K. y Eller, B. (2000). Psicología educativa para la enseñanza eficaz. México, D.F.: International Thomson Editores.

Hurtado, Albir, A., Galán-Mañas, A.; Kuznik, A. et al. [Grupo PACTE] (2019).

Establecimiento de niveles de competencias en traducción. Primeros resultados del proyecto NACT. Onomázein, 43, 1-25. DOI 10.7764/onomazein.43.08.

Hurtado Albir, A. (1996): "La enseñanza de la traducción directa 'general'. Objetivos de aprendizaje y metodología". En: Hurtado Albir, Amparo (ed.) La enseñanza de la traducción. Castellón: Universitat Jaume I, pp. 31-56.

Landeros Casillas, M. I. (2016). La identidad y el empoderamiento de las mujeres gambianas narrados a través de discursos fotográficos. Tercio reciente. Revista de Estudios en Sociedad, Artes y Gestión Cultural. 10, julio, 7-24. http://dx.doi.org/10.17561/rtc.n10.1

Lefton, L. y Brannon, L. (2006). Psychology. Pearson.

Martínez, N. (11 de mayo, 2006). El prototipo de la madre mexicana ya no existe: UNAM, El Universal. En línea https://archivo.eluniversal.com.mx/nacion/138255.html

Merriam Webster. (2021). Online dictionary (11th ed.). Recuperado de https://www.merriamwebster.com/dictionary/status?src=search-dict-hed

McCurdy, D.W., Spradley J. P. y Shandy, D. (2005). The Cultural Experience. Ethnography in Compex Society. Long Grove, Illinois: Waveland Press Inc.

Moon, U. J. y Bouchey, H. A. (2019). Effects of perceived parents' and teachers' values and beliefs on rural adolescents' college plans. The Social Science Journal, Official Journal of the Western Social Science Association, 56 (4), 458-469.

Muñoz Martín, R. (2008). Apuntes para una traductología cognitiva. En L. Pejenaute, J. Decesaris, M. Tricás, y E. Bernal (Eds.), Actas del III Congreso Internacional de la 
Asociación Ibérica de Estudios de Traducción e Interpretación. La traducción del futuro: Mediación lingüística y cultural en el siglo XXI. Barcelona, 22-24 de marzo de 2007. Vol.2 (pp.65-75). Barcelona: PPU.

Murray, S., O' Brien, M., y O' Campo, P. (2008). School Adjustment in the Early Grades: Toward an Integrated Model of Neighborhood, Parental, and Child Processes. Review of Educational Research, 78 (1), 3-32. http://rer.sagepub.com/content/78/1/3

Netquest (2020). Calculadora de muestras. Conoce el número de entrevistas que tienes que realizar. Disponible en https://bit.ly/30Rj0rl

Olk, H. (2009). Translation, Cultural Knowledge and Intercultural Competence. Journal of Intercultural Communication, 20, mayo. http://www.immi.se/intercultural/.

PACTE (2000). Acquiring Translation Competence: Hypotheses and Methodological Problems in a Research Project. En A., Beeby, D., Ensinger, y M., Presas (Ed.), Investigating Translation (pp. 99-106). Amsterdam: John Benjamins.

PACTE (2001). La competencia traductora y su adquisición. Quaderns. Revista de Traducció. 6, 39-45.

PACTE (2003). Building a Translation Competence Model. En F. Alves (Ed.), Triangulating Translation: Perspectives in Process Oriented Research (pp. 43-66). Amsterdam: John Benjamins.

PACTE (2005). Investigating Translation Competence: Conceptual and Methodological Issues. Meta: Translators' Journal, 50(2), 609-619. https://bit.ly/38YssKW.

PACTE (2008). First results of a Translation Competence Experiment: 'Knowledge of Translation' and 'Efficacy of the Translation Process.' En J., Kearns (Ed.), Translator and Interpreter Training. Issues, Methods and Debates (pp. 104-126). London: Continuum.

PACTE (2009). Results of the Validation of the PACTE Translation Competence Model: Acceptability and Decision Making, Across Languages and Cultures, 10 (2), 207-230. DOI: 10.1556/Acr.10.2009.2.3.

PACTE (2011). Results of the Validation of the PACTE Translation Competence Model: Translation Project and Dynamic Translation Index. En S., O’Brien (Ed.), IATIS Yearbook 2010 (pp. 609-619). London: Continuum.

PACTE (2014). First Results of PACTE Group's Experimental Research on Translation Competence Acquisition: The Acquisition of Declarative Knowledge of Translation en MonTI : Monografías de Traducción e Interpretación. DOI: https://doi.org/10.6035/http://dx.doi.org/10.6035/MonTI.2014.ne1.2

Pintrich, P. R., \& Schunk, D. H. (2002). Motivation in Education. Englewood Cliffs, NJ: Prentice Hall.

Pomerantz, E., Moorman, E., y Litwack, S. (2007) The How, Whom, and Why of Parents' Involvement in Children's Academic Lives: More Is Not Always Better. Review of Educational Research, 77 (3), 373 410, Recuperado de shorturl.at/rtuH6

Pozo, I. (2005). Aprendices y Maestros. La nueva cultura del aprendizaje. Madrid: Alianza Editorial. 
Pym, A. (1992). Translation and Text Transfer. An Essay on the Principles of Intercultural Communication, Frankfurt: Peter Lang.

Pym, A. (2003). Redefining Translation Competence in an Electronic Age. META Translators' Journal, 48 (4), 481-497. DOI: https://doi.org/10.7202/008533ar

Robinson, D. (1997). Becoming a translator. London/New York: Routledge.

Ryan, R. M. y Deci, E.L. (2000). Intrinsic and Extrinsic Motivations: Classic Definitions and New Directions. Contemporary Educational Psychology, 25, 54-67. https://bit.ly/30OQTsv

Sánchez, A., Reyes, F. y Villarroel, V. (2016). Participación y expectativas de los padres sobre la educación de sus hijos en una escuela pública. Estudios Pedagógicos, 42 (3),

347-367. http://dx.doi.org/10.4067/S0718-07052016000400019

Schmitt-Wilson, S. (2013). Social class and expectations of rural adolescents: The role of parental expectations. The Career Development Quarterly, 61, 226-239. https://doi.org/10.1002/j.21610045.2013.00051.x

Smith, L. (2000). La construcción social de la comprensión racional. En Tryphon, A. y Vonèche, J. (Comps.), Piaget-Vygotsky: La génesis social del pensamiento (pp. 143-166). Buenos Aires: Paidós.

Steven, J. Matthiesen, S. (1991). Preparing for the TOEFL, The Newbury House TOEFL Preparation Kit, Kennedy, D (Ed.), Thomson Learning EMEA, Limited.

Stevens, P. (2007). Researching Race/Ethnicity and Educational Inequality in EnglishSecondary Schools: A Critical Review of the Research Literature Between 1980 and 2005. Review of Educational Research, 72 (2), 147-185. https://bit.ly/3gXR1Mg

Wright, J. y Wiediger, V. (2007). Motivated Behaviors: The Interaction of Attention, Habituation and Memory. En L.V. Brown (Ed.), Psychology of Motivation (pp. 5-28) New York: Nova Science Publishers. 